\title{
Positive parity pentaquarks in a Goldstone boson exchange model
}

\author{
Fl. Stancu* \\ Université de Liège, Institut de Physique B.5, Sart Tilman, B-4000 Liège 1, Belgium
}

(September 21, 2021)

\begin{abstract}
We study the stability of the pentaquarks $u u d d \bar{Q}, u u d s \bar{Q}$ and $u d s s \bar{Q}(\mathrm{Q}=$ $\mathrm{c}, \mathrm{b}$, or t) of positive parity in a constituent quark model based on Goldstone boson exchange interaction between quarks. The pentaquark parity is the antiquark parity times that of a quark excited to a p-shell. We show that the Goldstone boson exchange interaction favors these pentaquarks much more than the negative parity ones of the same flavour content but all quarks in the ground state. We find that the nonstrange pentaquarks are stable against strong decays.
\end{abstract}

The existence of particles made of more than three quarks is an important issue of QCD inspired model. The interest has been raised so far by particles described by the colour state $[222]_{C}$, the tetraquarks $q^{2} \bar{q}^{2}$, the pentaquarks $q^{4} \bar{q}$ and the hexaquarks $q^{6}$. The present study is devoted to pentaquarks, first proposed independently by Gignoux, Silvestre-Brac and Richard [1] and Lipkin [2] about ten years ago. Within a constituent quark model based on one-gluon exchange (OGE) interaction, these authors found that the states $P_{\bar{c} s}^{0}=|u u d s \bar{c}\rangle$ and $P_{\bar{c} s}^{-}=|u d d s \bar{c}\rangle$ and their conjugates are stable against strong decays. Within better approximations, they turned out to be unstable [3, [4]. A systematic theoretical study [5] in a model with OGE interaction suggested several candidates for stability, and among them

\footnotetext{
*e-mail : fstancu@ulg.ac.be
} 
especially those with strangeness $\mathrm{S}=-1$ or -2 . In particular, the $u u d s \bar{c}$ system was bound by $-52 \mathrm{MeV}$. The nonstrange systems $u u d d \bar{Q}(\mathrm{Q}=\mathrm{c}$ or b) were unbound.

Calculations done within an instanton model [6] or a Skyrme model [7] show that pentaquarks, irrespective of their strangeness, appear as bound or near-threshold resonances depending on the model parameters. Moreover, the lowest pentaquark states predicted in Ref. [7] have positive parity $(\mathrm{L}=1)$.

If bound, the lifetime of the pentaquark $u u d s \bar{c}$ or $u d d s \bar{c}$ is expected to be similar to that of the $D_{s}^{ \pm}$meson. Using various mechanisms, the typically estimated pentaquark production cross section is of the order of $1 \%$ that of $D_{s}^{ \pm}[8]$. Based on the above theoretical predictions, experiments are being planned and the first search for the pentaquarks $P_{\bar{c} s}^{0}$ and $P_{\bar{c} s}^{-}$, performed at Fermilab, has just been reported [9]. The decay $P_{\bar{c} s}^{0} \rightarrow \phi \pi p$ was analyzed for two different pentaquark masses : $\mathrm{M}=2.75 \mathrm{GeV}$, being the lowest mass expected from the OGE model and $\mathrm{M}=2.86 \mathrm{GeV}$, the value at which the largest number of events was observed. No convincing evidence for pentaquarks decaying to $\phi \pi p$ was observed yet.

The theoretical predictions are definitely model-dependent. In a previous work [10], we studied the stability of heavy-flavoured pentaquarks within a chiral constituent quark model [11 [13] originally proposed by Glozman and Riska [11]. In this model, the hyperfine splitting in hadrons is obtained from the short-range part of the Goldstone boson exchange (GBE) interaction between quarks, instead of the OGE interaction of conventional models, as discussed above. The main merit of the GBE interaction is that it reproduces the correct ordering of positive and negative parity states in all parts of the considered spectrum [12 [14] in contrast to any other OGE model.

In Ref. [10], we considered pentaquarks with strangeness ranging from $S=-3$ to $S=$ 0. There, we assumed that all light quarks are identical and the ground-state orbital (O) wave function is symmetric under permutation of light quarks, i.e. it corresponds to the Young diagram $[4]_{O}$. The subsystem of light quarks must necessarily be in a colour 3, or alternatively a $[211]_{C}$ state. Then, the Pauli principle allows a certain number of spin $[f]_{S}$ and flavour $[f]_{F}$ states to be combined with $[4]_{O}$ and $[211]_{C}$ to give a total antisymmetric 
four-quark state. We found that any of these states combined with a heavy antiquark $\bar{Q}$ where $\mathrm{Q}=\mathrm{c}$ or $\mathrm{b}$ gave rise to a pentaquark energy which was at least 300-400 MeV above the dissociation threshold nucleon plus meson, i.e. the considered pentaquark cannot be a bound compact object. Its parity is $\mathrm{P}=-1$, due to the antiquark.

The novelty of this study is that, within the same GBE model, we analyse the stability of pentaquarks with $\mathrm{P}=+1$. In such a case, the parity of the pentaquark is given by $\mathrm{P}=(-)^{\mathrm{L}+1}$, thus the light quarks must carry an angular momentum L odd. Here, we consider the case $\mathrm{L}=1$, which implies that the subsystem of four light quarks must be in a state of orbital symmetry $[31]_{O}$. Although the kinetic energy of such a state is higher than that of the totally symmetric $[4]_{O}$ state, a schematic estimate [14 suggests that the $[31]_{O}$ symmetry would lead to a stable pentaquark. In the following, we give the arguments of Ref. [14 based on a simplified GBE interaction of the form

$$
V_{\chi}=-C_{\chi} \sum_{i<j} \lambda_{i}^{F} \cdot \lambda_{j}^{F} \vec{\sigma}_{i} \cdot \vec{\sigma}_{j}
$$

with $\lambda_{i}^{F}$ the Gell-Mann matrices, $\vec{\sigma}_{i}$ the Pauli matrices and $C_{\chi} \cong 30 \mathrm{MeV}$, determined from the $\Delta-\mathrm{N}$ splitting $[11$.

In the spirit of Glozman and Riska's model, there is no meson-exchange interaction between quarks and antiquarks. It is assumed that the $q \bar{q}$ pseudoscalar pair interaction is automatically included in the GBE interaction [11]. Then, as far as the spin-spin interaction is concerned, the discussion is restricted to the light $q^{4}$ subsystem. The Pauli principle allows for the following two totally antisymmetric states with $[31]_{O}$ symmetry, written in the flavour-spin (FS) coupling scheme

$$
\begin{aligned}
& |1\rangle=\left([31]_{O}[211]_{C}\left[1^{4}\right]_{O C} ;[22]_{F}[22]_{S}[4]_{F S}\right) \\
& |2\rangle=\left([31]_{O}[211]_{C}\left[1^{4}\right]_{O C} ;[31]_{F}[31]_{S}[4]_{F S}\right)
\end{aligned}
$$

Asymptotically, a ground state baryon and a meson, into which a pentaquark can split, would give $[3]_{O} \times[2]_{O}=[5]_{O}+[41]_{O}+[32]_{O}$. By removing the antiquark, one can make 
the reduction $[41]_{O} \rightarrow[31]_{O} \times[1]_{O}$ or $[32]_{O} \rightarrow[31]_{O} \times[1]_{O}$. Thus, the symmetry $[31]_{O}$ of the light quark subsystem is compatible with an $\mathrm{L}=1$ asymptotically separated baryon plus meson system.

The expectation value of (1) calculated, for example, according to the Appendix of Ref. [15], is $-28 C_{\chi}$ for $|1\rangle$ and $-64 / 3 C_{\chi}$ for $|2\rangle$. These two states would actually couple via a quark-antiquark spin-spin interaction to a total angular momentum $\mathrm{J}=\frac{1}{2}$ or $\frac{3}{2}$ where $\vec{J}=\overrightarrow{\mathrm{L}}+\overrightarrow{\mathrm{S}}+\overrightarrow{\mathrm{s}}_{Q}$, with $\overrightarrow{\mathrm{L}}, \overrightarrow{\mathrm{S}}$ the angular momentum and spin of the light system and $\vec{s}_{Q}$ the spin of the antiquark. As the quark-antiquark interaction is neglected here, in the following we restrict our discussion to the lowest state, i.e. $|1\rangle$. The quark-antiquark interaction is neglected in the description of mesons as well, as for example in Ref. [16], so that the meson Hamiltonian contains a kinetic and a confinement term only.

We are interested in the quantity

$$
\Delta E=E\left(q^{4} \bar{Q}\right)-E\left(q^{3}\right)-E(q \bar{Q})
$$

In our schematic estimate, we suppose that the confinement energy roughly cancels out in $\Delta E$. Then, the kinetic energy contribution $\Delta K E$ to $\Delta E$ is $\Delta K E=5 / 4 \hbar \omega$ in a harmonic oscillator model and the GBE contribution for the state $|1\rangle$ is $\Delta V_{\chi}=-14 C_{\chi}$. With $\hbar \omega \approx 250 \mathrm{MeV}$, determined from the $\mathrm{N}(1440)$ - $\mathrm{N}$ splitting [11], this would give

$$
\Delta E=\frac{5}{4} \hbar \omega-14 C_{\chi}=-107.5 \mathrm{MeV}
$$

i.e. a substantial binding. This is to be contrasted with the negative parity pentaquarks studied in Ref. [10] where for the lowest state one has $\Delta E=3 / 4 \hbar \omega-2 C_{\chi}=127.5 \mathrm{MeV}$, i.e. unstability, consistent with the detailed study made in [10].

The estimate (5) is a consequence of the flavour dependence of the GBE interaction. For a specific spin state $[f]_{S}$, a schematic OGE interaction of type $V_{c m}=-C_{c m} \sum \lambda_{i}^{c} \cdot \lambda_{j}^{c} \vec{\sigma}_{i} \cdot \vec{\sigma}_{j}$ does not make a distinction between $[4]_{O}$ and $[31]_{O}$ so that the $[31]_{O}$ state will appear higher than $[4]_{O}$ due to the kinetic energy. The GBE interaction overcomes the excess of kinetic contribution in $[31]_{O}$ and generates a lower expectation value for $[31]_{O}$ than for $[4]_{O}$. 
The GBE Hamiltonian has the form 12$]$ :

$$
H=\sum_{i} m_{i}+\sum_{i} \frac{\vec{p}_{i}^{2}}{2 m_{i}}-\frac{\left(\sum_{i} \vec{p}_{i}\right)^{2}}{2 \sum_{i} m_{i}}+\sum_{i<j} V_{\text {conf }}\left(r_{i j}\right)+\sum_{i<j} V_{\chi}\left(r_{i j}\right)
$$

with the linear confining interaction :

$$
V_{\text {conf }}\left(r_{i j}\right)=-\frac{3}{8} \lambda_{i}^{c} \cdot \lambda_{j}^{c} C r_{i j}
$$

and the spin-spin component of the GBE interaction in its $S U_{F}(3)$ form :

$$
\begin{aligned}
V_{\chi}\left(r_{i j}\right) & =\left\{\sum_{F=1}^{3} V_{\pi}\left(r_{i j}\right) \lambda_{i}^{F} \lambda_{j}^{F}\right. \\
& \left.+\sum_{F=4}^{7} V_{K}\left(r_{i j}\right) \lambda_{i}^{F} \lambda_{j}^{F}+V_{\eta}\left(r_{i j}\right) \lambda_{i}^{8} \lambda_{j}^{8}+V_{\eta^{\prime}}\left(r_{i j}\right) \lambda_{i}^{0} \lambda_{j}^{0}\right\} \vec{\sigma}_{i} \cdot \vec{\sigma}_{j},
\end{aligned}
$$

with $\lambda^{0}=\sqrt{2 / 3} \mathbf{1}$, where 1 is the $3 \times 3$ unit matrix. The interaction (8) contains $\gamma=\pi, K, \eta$ and $\eta^{\prime}$ meson-exchange terms and the form of $V_{\gamma}\left(r_{i j}\right)$ is given as the sum of two distinct contributions : a Yukawa-type potential containing the mass of the exchanged meson and a short-range contribution of opposite sign, the role of which is crucial in baryon spectroscopy. For a given meson $\gamma=\pi, K, \eta$ or $\eta^{\prime}$, the meson exchange potential is

$$
V_{\gamma}(r)=\frac{g_{\gamma}^{2}}{4 \pi} \frac{1}{12 m_{i} m_{j}}\left\{\theta\left(r-r_{0}\right) \mu_{\gamma}^{2} \frac{e^{-\mu_{\gamma} r}}{r}-\frac{4}{\sqrt{\pi}} \alpha^{3} \exp \left(-\alpha^{2}\left(r-r_{0}\right)^{2}\right)\right\}
$$

For the Hamiltonian (6)-(9), we use the parameters of Refs. [12,13]. These are :

$$
\begin{gathered}
\frac{g_{\pi q}^{2}}{4 \pi}=\frac{g_{\eta q}^{2}}{4 \pi}=\frac{g_{K q}^{2}}{4 \pi}=0.67, \frac{g_{\eta^{\prime} q}^{2}}{4 \pi}=1.206 \\
r_{0}=0.43 \mathrm{fm}, \alpha=2.91 \mathrm{fm}^{-1}, C=0.474 \mathrm{fm}^{-2}, m_{u, d}=340 \mathrm{MeV}, m_{s}=440 \mathrm{MeV} \\
\mu_{\pi}=139 \mathrm{MeV}, \mu_{\eta}=547 \mathrm{MeV}, \mu_{\eta^{\prime}}=958 \mathrm{MeV}, \mu_{K}=495 \mathrm{MeV} .
\end{gathered}
$$

The masses of the threshold hadrons are calculated variationally as in Ref. [10] where we assume an $s^{3}$ configuration for baryons. They are given in Table 1 where a theoretical $\bar{t} s$ meson is also included in order to analyse the large $m_{Q}$ limit. The mass of the $\bar{t} s$ meson has been determined by taking $m_{t}=175 \mathrm{GeV}$.

For pentaquarks, we used the internal Jacobi coordinates 


$$
\begin{gathered}
\vec{x}=\vec{r}_{1}-\vec{r}_{2}, \quad \vec{y}=\left(\vec{r}_{1}+\vec{r}_{2}-2 \vec{r}_{3}\right) / \sqrt{3} \\
\vec{z}=\left(\vec{r}_{1}+\vec{r}_{2}+\vec{r}_{3}-3 \vec{r}_{4}\right) / \sqrt{6}, \quad \vec{t}=\left(\vec{r}_{1}+\vec{r}_{2}+\vec{r}_{3}+\vec{r}_{4}-4 \vec{r}_{5}\right) / \sqrt{10}
\end{gathered}
$$

First, we expressed the $q^{4}$ orbital wave functions of symmetry $[31]_{O}$ in terms of the above Jacobi coordinates. We assumed an $s^{3} p$ structure for $[31]_{O}$ and inspired by Moshinski's method [17], we found the content of the three independent [31] $]_{O}$ states [18], denoted below by $\psi_{i}$, in terms of shell model functions $|n \ell m\rangle$. The result is

$$
\begin{aligned}
& \psi_{1}=\begin{array}{|l|l|l|}
\hline 1 & 2 & 3 \\
\hline 4 &
\end{array}=\langle\vec{x} \mid 000\rangle\langle\vec{y} \mid 000\rangle\langle\vec{z} \mid 001\rangle
\end{aligned}
$$

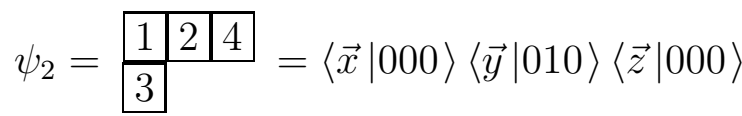

$$
\begin{aligned}
& \psi_{3}=\begin{array}{|l|l|l}
\hline 1 & 3 & 4 \\
\hline 2 &
\end{array}=\langle\vec{x} \mid 010\rangle\langle\vec{y} \mid 000\rangle\langle\vec{z} \mid 000\rangle
\end{aligned}
$$

where, for convenience, we took the quantum number $\mathrm{m}=0$ everywhere. The pentaquark orbital wave functions $\psi_{i}^{5}$ are then obtained by multiplying each $\psi_{i}$ by the wave function $\langle\vec{t} \mid 000\rangle$ which describes the relative motion of the $q^{4}$ subsystem and the antiquark $\bar{Q}$. Assuming two variational parameters, $a$ for the internal motion of $q^{4}$ and $b$ for the relative motion of $q^{4}$ and $\bar{Q}$, we have explicitly

$$
\begin{aligned}
& \psi_{1}^{5}=N \exp \left[-\frac{a}{2}\left(x^{2}+y^{2}+z^{2}\right)-\frac{b}{2} t^{2}\right] z Y_{10}(\hat{z}) \\
& \psi_{2}^{5}=N \exp \left[-\frac{a}{2}\left(x^{2}+y^{2}+z^{2}\right)-\frac{b}{2} t^{2}\right] y Y_{10}(\hat{y}) \\
& \psi_{3}^{5}=N \exp \left[-\frac{a}{2}\left(x^{2}+y^{2}+z^{2}\right)-\frac{b}{2} t^{2}\right] x Y_{10}(\hat{x})
\end{aligned}
$$

where 


$$
N=\frac{2^{3 / 2} a^{11 / 4} b^{3 / 4}}{3^{1 / 2} \pi^{5 / 2}}
$$

The kinetic energy part of (6) can be calculated analytically. For the state (2) or (3), its form is

$$
\begin{aligned}
\langle T\rangle & =\frac{1}{3}\left[\left\langle\psi_{1}^{5}|T| \psi_{1}^{5}\right\rangle+\left\langle\psi_{2}^{5}|T| \psi_{2}^{5}\right\rangle+\left\langle\psi_{3}^{5}|T| \psi_{3}^{5}\right\rangle\right] \\
& =\hbar^{2}\left(\frac{11}{2 \mu_{1}} a+\frac{3}{2 \mu_{2}} b\right)
\end{aligned}
$$

with

$$
\frac{4}{\mu_{1}}=\left\{\begin{array}{l}
\frac{1}{m_{1}}+\frac{3}{m_{2}} \text { for } q_{1} q_{2}^{3} \\
\frac{2}{m_{1}}+\frac{2}{m_{2}} \text { for } q_{1}^{2} q_{2}^{2}
\end{array}\right.
$$

where $q_{1}, q_{2}$ are light quarks and

$$
\frac{5}{\mu_{2}}=\frac{1}{\mu_{1}}+\frac{4}{m_{Q}}
$$

$m_{Q}$ representing the heavy antiquark mass. Here, we choose $m_{c}=1.35 \mathrm{GeV}, m_{b}=4.66$ GeV according to Ref. [10] and $m_{t}=175 \mathrm{GeV}$. Taking $m_{u}=m_{d}=m_{s}=m_{Q}$ and setting $\mathrm{a}=\mathrm{b}$, the identical particle limit $\langle T\rangle=\frac{7}{2} \hbar \omega$ with $\hbar \omega=2 a \hbar^{2} / \mathrm{m}$ is recovered correctly.

Integrating in the coulour space as shown in Ref. [10], the confinement part of (6) becomes

$$
\left\langle V_{\text {conf }}\right\rangle=\frac{C}{2}\left(6\left\langle r_{12}\right\rangle+4\left\langle r_{45}\right\rangle\right)
$$

where the coefficients 6 and 4 account for the number of light-light and light-heavy pairs, respectively, and

$$
\left\langle r_{i j}\right\rangle=\frac{1}{3}\left[\left\langle\psi_{1}^{5}\left|r_{i j}\right| \psi_{1}^{5}\right\rangle+\left\langle\psi_{2}^{5}\left|r_{i j}\right| \psi_{2}^{5}\right\rangle+\left\langle\psi_{3}^{5}\left|r_{i j}\right| \psi_{3}^{5}\right\rangle\right]
$$

An analytic evaluation gives

$$
\left\langle r_{12}\right\rangle=\frac{20}{9} \sqrt{\frac{1}{\pi a}}
$$


and

$$
\left\langle r_{45}\right\rangle=\frac{1}{3 \sqrt{2 \pi}}\left[2 \sqrt{\frac{3}{a}+\frac{5}{b}}+\sqrt{5 b}\left(\frac{1}{2 a}+\frac{1}{b}\right)\right]
$$

The matrix elements of the spin-flavour operators of (8) have been calculated using the fractional parentage technique described in Ref. [18] based on Clebsch-Gordan coefficients of the group $S_{4}$ [19]. In this way, each matrix element reduces to a linear combination of twobody matrix elements of either symmetric or antisymmetric states for which Eqs. (3.3) of Ref. [11] can be used to integrate in the spin-flavour space. The resulting linear combinations contain orbital two-body matrix elements of type $\left\langle s s\left|V_{\gamma}\right| s s\right\rangle,\left\langle s p\left|V_{\gamma}\right| s p\right\rangle$ and $\left\langle s p\left|V_{\gamma}\right| p s\right\rangle$ where $\gamma=\pi, K, \eta$ and $\eta^{\prime}$ as in Eq. (8).

In Table 2, we present results for $S=0, S=-1$ and $S=-2$ pentaquarks. The quantity $\Delta E$, defined by (4) and exhibited in column 5, is obtained from $E\left(q^{4} \bar{Q}\right)$ calculated variationally for the state $|1\rangle$ defined by (2). The optimal values of the parameters $a$ and $b$ are indicated in each case. In all cases, one has $a>b$. The inverses $1 / a$ and $1 / b$ give an idea of the quark-quark and quark-antiquark distances, respectively. One can see that at equilibrium the light quarks are clustered together, orbitting around the heavy antiquark.

The present variational solution does not give binding for $u u d s \bar{Q}$ and $u d s s \bar{Q}$. The quantity $\Delta E$ decreases smoothly with increasing $m_{Q}$ but does not become negative even for $m_{t}=175 \mathrm{GeV}$. However, as expected from the discussion around Eq. (5), there is much less "repulsive" effect in these type of pentaquarks than that appearing for the negative parity pentaquarks studied in Ref. [10], where $\Delta E \sim 370-490 \mathrm{MeV}$ for pentaquarks with similar flavour content.

But the nonstrange positive parity pentaquarks $u$ udd $\bar{Q}$ are bound by $-75.6 \mathrm{MeV},-95.6$ $\mathrm{MeV}$ and $-102.8 \mathrm{MeV}$ for $\mathrm{Q}=\mathrm{c}, \mathrm{b}$ and $\mathrm{t}$, respectively. The reason is that the GBE interaction is stronger in the nonstrange case because of the $1 / m_{i} m_{j}$ factor in Eq. (9). Thus, the GBE model suggests that the nonstrange positive parity pentaquarks are the best candidates for stable compact systems. This in contrast to OGE based models where strangeness is required [5] in order to reach stability for heavy-flavoured pentaquarks. The present results 
have similarities with those obtained in [7] from the Skyrme model : 1) the lowest pentaquark states have positive parity for any flavour content ; 2) stability does not necessarily require strangeness.

From Table 2 we can obtain upper limits for the masses of the stable quarks as $M(u u d d \bar{c})$ $=2.895 \mathrm{GeV}$ and $M(u u d d \bar{b})=6.176 \mathrm{GeV}$.

It would be very useful indeed to look for a better variational solution for positive parity pentaquarks to see whether the soft repulsion persists or disappears for strange systems and to improve the upper bounds for the masses of the stable states. A possible way would be to consider interference effects with other asymptotic channels, as for example, in Ref. [3]. It would be also interesting to see how the binding energy of the pentaquarks depends on the particular parametrization of the hyperfine interaction (nonrelativistic [12,13] versus semi-relativistic [21] GBE model).

Irrespective of the type of hyperfine interaction, an important issue remains the model of confinement. Both in OGE based calculations [2 5] and here, the confinement relies on a $\lambda_{i}^{c} \cdot \lambda_{j}^{c}$ ansatz, which does not respect local colour gauge invariance. Despite this defect, the first estimates have been done under this assumption, for simplicity. For baryons, the ansatz $\lambda_{i}^{c} \cdot \lambda_{j}^{c}$ is a good approximation and it remains to be seen how good it is for pentaquarks.

But, at any level of accuracy, it is expected that the upper bound obtained for pentaquarks of negative parity, as those studied in Ref. [10], would remain higher than the upper bound obtained for positive parity pentaquarks as introduced here. In an OGE based model, the situation will be the other way round. It remains to future experiments to disentangle between GBE and OGE models, through the search of pentaquarks.

\section{ACKNOWLEDGMENTS}

I am most grateful to Leonid Glozman for several useful comments, and to Dan Olof Riska for pointing out some features of the Skyrme model results. 


\section{REFERENCES}

[1] C. Gignoux, B. Silvestre-Brac and J.-M. Richard, Phys.Lett. B193 (1987) 323.

[2] H.J. Lipkin, Phys.Lett. B195 (1987) 484.

[3] S. Fleck, C. Gignoux, J.-M. Richard and B. Silvestre-Brac, Phys.Lett. B220 (1989) 616.

[4] S. Zouzou and J.-M. Richard, Few-Body Syst. 16 (1994) 1.

[5] J. Leandri and B. Silvestre-Brac, Phys.Rev. D40 (1989) 2340.

[6] S. Takeuchi, S. Nussinov and K. Kubodera, Phys.Lett. B318 (1993) 1.

[7] D.O. Riska and N.N. Scoccola, Phys. Lett. B299 (1993) 338.

[8] see e.g. M.A. Moinester, D. Ashery, L.G. Landsberg and H.J. Lipkin, Z.Phys. A356 (1996) 207.

[9] E.M. Aitala et al., Fermilab E791 Collaboration, preprint hep-ex/9709013.

[10] M. Genovese, J.-M. Richard, Fl. Stancu and S. Pepin, e-print hep-ph/9712452, Phys.Lett. B, to be published.

[11] L.Ya. Glozman and D.O. Riska, Phys. Rep. 268 (1996) 263.

[12] L.Ya. Glozman, Z. Papp and W. Plessas, Phys. Lett. B381 (1996) 311.

[13] L.Ya. Glozman, Z. Papp, W. Plessas, K. Varga and R.F. Wagenbrunn, Nucl. Phys. A623 (1997) 90c.

[14] L.Ya. Glozman and D.O. Riska, private communication.

[15] Fl. Stancu, S. Pepin and L.Ya. Glozman, Phys.Rev. C56 (1997) 2779.

[16] S. Pepin, Fl. Stancu, M. Genovese and J.-M. Richard, Phys. Lett. B393 (1997) 119.

[17] M. Moshinski, The Harmonic Oscillator in Modern Physics : from Atoms to Quarks, Gordon and Breach Science Publishers, New York 1969, Chapter 4. 
[18] Fl. Stancu, Group Theory in Subnuclear Physics, Clarendon Press, Oxford 1996, Chapters 4 and 10 .

[19] S. Pepin and Fl. Stancu, Isoscalar factor of the permutation group, preprint University of Liège, 1996 (unpublished).

[20] Particle Data Group, Phys.Rev. D54 (1996) 1.

[21] L.Ya. Glozman, W. Plessas, K. Varga and R.F. Wagenbrun, e-print hep-ph/9706507. 


\section{TABLES}

TABLE I. Masses of hadrons required to calculate the threshold energy $E_{T}=E_{\text {baryon }}+E_{\text {meson }}$. The experimental mass for mesons represents the average $\bar{M}=\left(M+3 M^{*}\right) / 4$, when both the pseudoscalar $M$ and the vector $M^{*}$ mesons masses are available [20] .

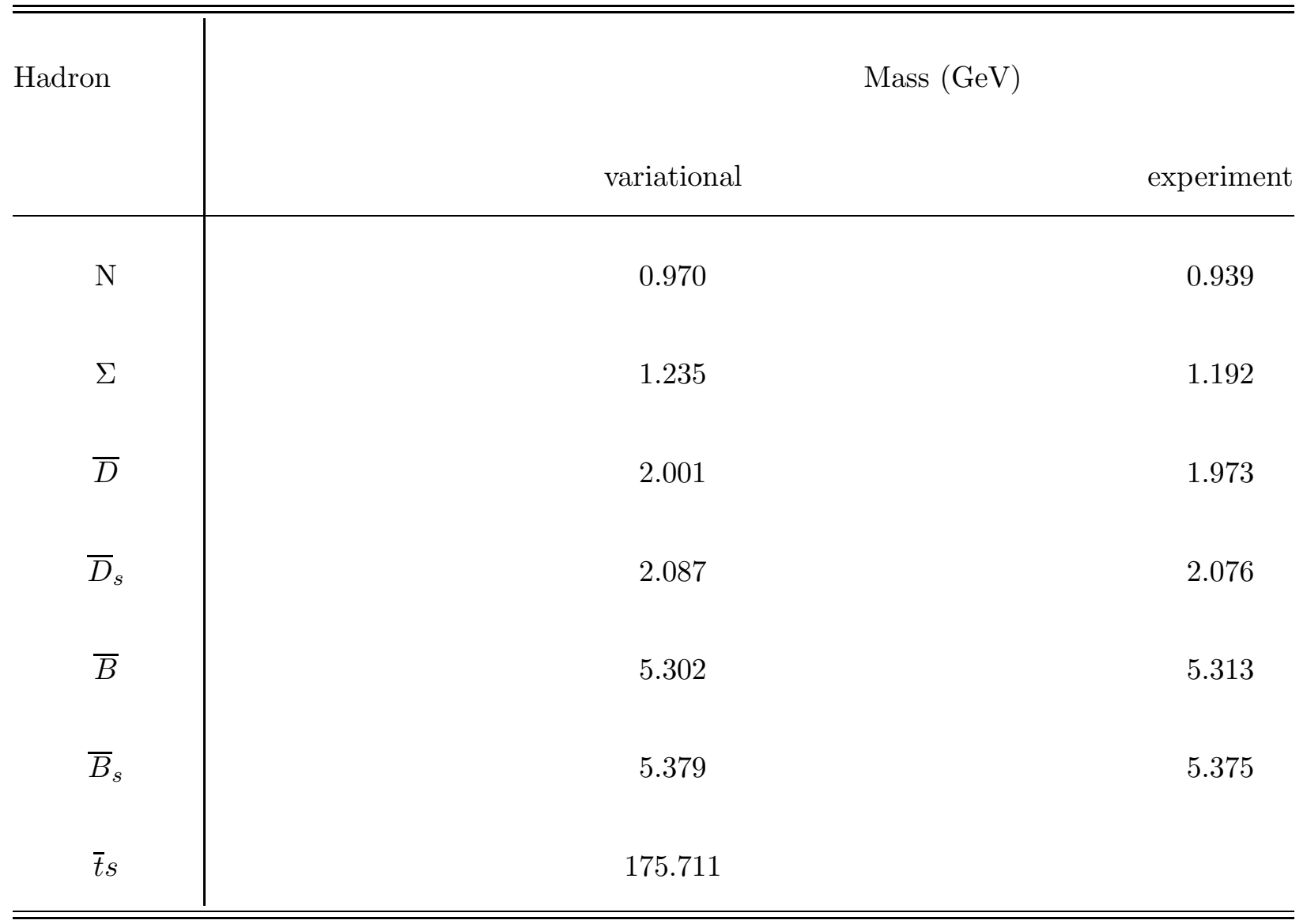


TABLE II. Lowest positive parity pentaquarks of total angular momentum $\mathrm{J}=1 / 2,3 / 2$ and symmetry structure given by Eq. (2). Column 1 gives the flavour content, column 2 the isospin I, columns 3 and 4 the optimal variational parameters associated to the wave functions (15)-(18), column 5 gives $\Delta E=E-E_{T}$ where $E$ is the upper bound for the expectation value of the Hamiltonian (6)-(10) and $E_{T}=E_{\text {baryon }}+E_{\text {meson }}$ (see Table 1) for the threshold given in the last column.

\begin{tabular}{|c|c|c|c|c|c|}
\hline \multirow[t]{2}{*}{ Pentaquark } & \multirow[t]{2}{*}{$\mathrm{I}$} & \multicolumn{2}{|c|}{ Variational parameters $\left(G e V^{2}\right)$} & \multirow{2}{*}{$\begin{array}{c}\Delta E \\
(\mathrm{MeV})\end{array}$} & \multirow[t]{2}{*}{ Threshold } \\
\hline & & a & $\mathrm{b}$ & & \\
\hline$u u d d \bar{c}$ & 0 & 0.110 & 0.040 & -75.6 & $N+\bar{D}$ \\
\hline$u u d d \bar{b}$ & 0 & 0.110 & 0.053 & -95.6 & $N+\bar{B}$ \\
\hline$u u d d \bar{t}$ & 0 & 0.110 & 0.061 & -102.8 & $N+\bar{t} s$ \\
\hline$u u d s \bar{c}$ & $1 / 2$ & 0.101 & 0.041 & 104.7 & $N+\bar{D}_{s}$ \\
\hline$u u d s \bar{b}$ & $1 / 2$ & 0.102 & 0.054 & 93.4 & $N+\bar{B}_{s}$ \\
\hline$u u d s \bar{t}$ & $1 / 2$ & 0.102 & 0.063 & 86.9 & $N+\bar{t} s$ \\
\hline$u d s s \bar{c}$ & 1 & 0.092 & 0.041 & 81.0 & $\Sigma+\bar{D}_{s}$ \\
\hline$u d s s \bar{b}$ & 1 & 0.092 & 0.055 & 69.3 & $\Sigma+\bar{B}_{s}$ \\
\hline$u d s s \bar{t}$ & 1 & 0.093 & 0.064 & 62.6 & $\Sigma+\bar{t} s$ \\
\hline
\end{tabular}

